\title{
Don't stress and repair the damage
}

Uropathogenic Escherichia coli (UPEC) is the most common causative agent for urinary tract infections. It has been proposed that a subpopulation of persister cells initiate recurrent infections; however, the molecular mechanisms involved in the formation of persister cells was elusive. Here, Molina-Quiroz et al. investigated persister cell formation in exponentially growing UPEC cultures that were exposed to different antibiotics. They showed that persistence involves decreased levels of reactive oxygen species and SOSdependent DNA repair and that these processes are regulated by the second messenger cAMP.

The authors first reported that UPEC mutants that are deficient in cAMP synthesis ( $\triangle c y a A$ mutants) exhibited increased survival following exposure to cell wall-acting antibiotics compared with wild-type bacteria. However, they did not observe a difference in survival rate when UPEC cells were exposed to gentamicin or a decreased survival rate compared with wild-type cells when UPEC cells were exposed to ciprofloxacin. Addition of exogenous cAMP decreased the survival rates of $\triangle c y a A$ mutant cells to levels that were similar to those observed for the wild-type cells. Moreover, exponentially growing cell cultures in which the cAMP receptor protein (CRP) was deleted also exhibited higher survival rates in the presence of ampicillin than wild-type cultures; however, the addition of exogenous cAMP did not reduce the survival of $\Delta c r p$ mutants. The data are consistent with the hypothesis that cAMP-CRP has an inhibitory effect on the formation of persisters in UPEC cultures and that this role is likely to be dependent on the antibiotic target.

Next, the authors constructed transposon libraries in the wildtype and $\triangle c y a A$ backgrounds and screened for mutants with decreased survival following exposure to ampicillin. For $\triangle c y a A$ mutants, they found that genes associated with oxidative stress contribute to survival, whereas the screen revealed genes mainly involved in cell envelope biogenesis to be important in the wild-type cells. Indeed, mutating genes involved in the oxidative stress response in $\triangle c y a A$ mutants decreased survival rates, an effect that was not seen in the wild-type cells. The authors noted that more than one-third of the genes identified in the mutant screen in the $\triangle c y a A$ background contain a CRPbinding motif in their promoters; this is in agreement with the hypothesis that the oxidative stress response is induced in cultures lacking $c y a A$ and $c r p$ by the derepression of genes involved in this pathway. Indeed, the authors were able to show that oxidative stress responses are sufficiently induced in $\triangle c y a A$ mutant cells to detoxify reactive oxygen species that are generated in the presence of the antibiotic. Thus, the lack of cAMP seems to increase the ability of the bacteria to cope with oxidative damage.

Finally, it was previously reported that oxidative stress induces SOSdependent DNA repair and that the SOS response is regulated by

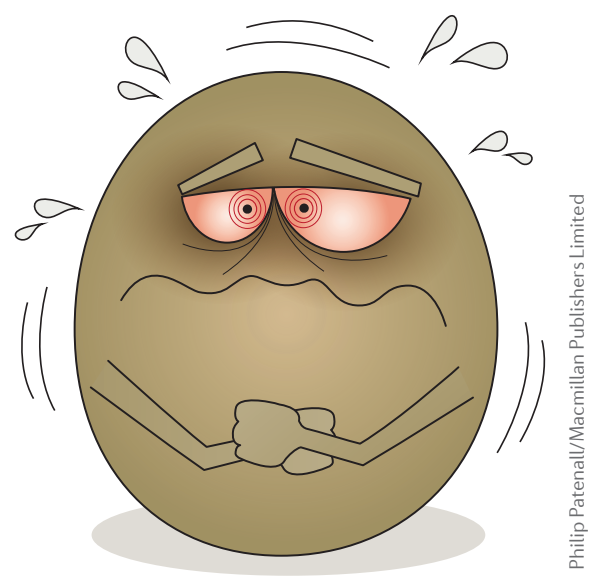

cAMP. Here, the authors found that a $\triangle c y a A$ mutant that lacks the SOS pathway exhibited lower survival rates following exposure to ampicillin compared with $\triangle c y a A$ mutants with a functional SOS response. Thus, the SOS pathway is required for the survival of the $\triangle c y a A$ mutant in the presence of the antibiotic.

In summary, the findings of the study suggest that the mechanisms of UPEC persistence in the presence of cell wall-acting antibiotics involve the induction of the oxidative damage response and DNA damage repair by the SOS pathway, and that these processes are regulated by cAMP in UPEC.

Andrea Du Toit

ORIGINAL ARTICLES Molina-Quiroz, R. C. et al. Cyclic AMP regulates bacterial persistence through repression of the oxidative stress response and SOS-dependent DNA repair in uropathogenic Escherichia coli. mBio 9, e02144-17 (2018) FURTHER READING Fisher, R. A., Gollan, B. \& Helaine, S. Persistent bacterial infections and persister cells. Nat. Rev. Microbiol. 15, 453-464 (2017) 